\title{
Lactobacillus gasseri SBT2055 inhibits adipose tissue inflammation and intestinal permeability in mice fed a high-fat diet
}

\author{
Michio Kawano*, Masaya Miyoshi, Akihiro Ogawa, Fumihiko Sakai and Yukio Kadooka \\ Milk Science Research Institute, Megmilk. Snow Brand Co. Ltd, 1-1-2 Minamidai, Kawagoe, Saitama, Japan \\ (Received 10 March 2016 - Accepted 18 March 2016)
}

Journal of Nutritional Science (2016), vol. 5, e23, page 1 of 9

doi:10.1017/jns.2016.12

Abstract

The probiotic Lactobacillus gasseri SBT2055 (LG2055) has anti-obesity effects. Obesity is closely correlated with inflammation in adipose tissue, and maintaining adipose tissue in a less-inflamed state requires intestinal integrity or a barrier function to protect the intestine from the disruption that can be caused by a high-fat diet (HFD). Here, we examined the anti-inflammatory and intestinal barrier-protecting effects of LG2055 in C57BL/6 mice fed a normal-fat diet (NFD), HFD, or the HFD containing LG2055 (HFD-LG) for 21 weeks. HFD-LG intake significantly prevented HFD-induced increases in body weight, visceral fat mass, and the ratio of inflammatory-type macrophages to anti-inflammatory ones in adipose tissue. Mice fed the HFD showed higher intestinal permeability to a fluorescent dextran administered by oral administration and an elevated concentration of antibodies specific to lipopolysaccharides (LPS) in the blood compared with those fed the NFD, suggesting an increased penetration of the gut contents into the systemic circulation. These elevations of intestinal permeability and anti-LPS antibody levels were significantly suppressed in mice fed the HFD-LG. Moreover, treatment with LG2055 cells suppressed an increase in the cytokine-induced permeability of Caco-2 cell monolayers. These results suggest that LG2055 improves the intestinal integrity, reducing the entry of inflammatory substances like LPS from the intestine, which may lead to decreased inflammation in adipose tissue.

Key words: Lactobacillus gasseri SBT2055: Intestinal barrier function: Anti-obesity effects: Anti-inflammation effects: Diet-induced obesity

Obesity is often associated with chronic low-grade inflammation that is a cause of insulin resistance that may in turn cause metabolic disorders ${ }^{(1)}$, in which pro-inflammatory cytokines, such as TNF- $\alpha$ and IL- 6 , inhibit insulin signalling in the muscle, liver and other organs ${ }^{(2)}$. In addition to pro-inflammatory cytokine expression, infiltration of macrophages and lymphocytes into adipose tissue is also critical for low-grade inflammation $^{(2)}$.

Mechanisms by which obesity-associated low-grade inflammation is initiated in adipose tissue remain controversial. Recent studies showed that lipopolysaccharides (LPS) derived from Gram-negative bacteria in the gut could be a trigger for adipose tissue inflammation and insulin resistance in obesity $^{(3,4)}$. In a healthy intestine, the translocation of LPS from the gut lumen into the circulation is prevented by the intestinal epithelial barrier function. However, once the intestinal barrier is disrupted under conditions such as alcohol intake ${ }^{(5)}$, dextran sodium sulfate treatment ${ }^{(6)}$, or restraint stress ${ }^{(7)}$, LPS levels in the blood increase. Cani et al. reported that a high-fat diet or an obese state disrupted the intestinal barrier and increased LPS levels in the blood $^{(4)}$. Furthermore, they demonstrated that mice administered LPS exhibited fat pad weight gain, inflammation in visceral adipose tissue and insulin resistance, all of which are common in obesity ${ }^{(3)}$. In human studies, the plasma LPS concentration is positively correlated with visceral fat volume and indexes of insulin

Abbreviations: FBS, fetal bovine serum; FCM, flow cytometry buffer; FD-4, fluorescein isothiocyanate-dextran; FITC, fluorescein isothiocyanate; HFD, high-fat diet; HFDLG, high-fat diet containing Lactobacillus gasseri SBT2055; IFN- $\gamma$, interferon- $\gamma$; LPS, lipopolysaccharide; LY, Lucifer yellow; M1, classically activated macrophages; M2, alternatively activated macrophages; NFD, normal-fat diet; SVF, stromal-vascular fraction; TEER, trans-epithelial electrical resistance.

* Corresponding author: M. Kawano, fax +8149242 8157, email michio-kawano@meg-snow.com 
resistance (for example, homeostatic model assessment of insulin resistance (HOMA-IR) index and HbA1c) ${ }^{(8,9)}$. Therefore, it is conceivable that the intestinal barrier disruption caused by HFD intake or obesity induces the entry of LPS into the circulation, leading to adipose tissue inflammation and insulin resistance.

Lactobacillus gasseri SBT2055 (LG2055) is a probiotic bacterium of human origin that has bile acid tolerance and can colonise the intestine and improve the intestinal environment ${ }^{(10-12)}$. In addition, LG2055 has anti-obesity effects; for example, LG2055-containing fermented milk and LG2055 cells themselves prevented the enlargement of adipocytes and an increase in abdominal fat volume in rats, mice and humans ${ }^{(13-16)}$. Furthermore, LG2055 improved the inflammatory status of the body and adipose tissue: fermented milk containing LG2055 prevented the elevation of the blood level of soluble intercellular adhesion molecule-1 (sICAM-1), a circulatory inflammatory marker, in rats fed a $10 \%$ fat $\operatorname{diet}^{(17)}$. LG2055 cells also prevented an increase in the mRNA expression of chemokine CC motif ligand 2 (CCL2) in adipose tissue, which contributes to macrophage infiltration into the tissue, in mice fed a $10 \%$ fat $\operatorname{diet}^{(15)}$. These results suggest that LG2055 has the ability to ameliorate systemic and adipose tissue inflammation in obesity. However, it has not been determined if LG2055 prevents the intestinal barrier disruption that contributes to the development of adipose tissue inflammation in obesity.

In the present study, we examined the anti-inflammatory and intestinal barrier-protecting effects of LG2055 in mice fed a high-fat $(20 \%(\mathrm{w} / \mathrm{w})$ fat $)$ diet containing LG2055 cells. We also used an intestinal epithelial cell culture model whose permeability was enhanced by inflammatory cytokines to determine whether LG2055 prevents the permeability.

\section{Experimental methods}

\section{Preparation of LG2055 cells}

LG2055 is a bacterial strain deposited in the International Patent Organism Depository, National Institute of Advanced Industrial Science and Technology (Tsukuba, Japan). LG2055 was cultured in a sterile de Man, Rogosa and Sharpe (MRS) broth (BD Bioscience) at $37^{\circ} \mathrm{C}$ for $16 \mathrm{~h}$. After cultivation, bacterial cells were collected by centrifugation at $4620 \mathrm{~g}$ for $10 \mathrm{~min}$ at $4{ }^{\circ} \mathrm{C}$, and washed twice with saline and once with sterile water. To prepare the experimental diet for the mice, LG2055 cells were suspended in a $10 \%(\mathrm{w} / \mathrm{w})$ lactose solution to reduce the loss of cell viability before lyophilisation. The lyophilised powder was weighed and the net LG2055 weight was calculated by subtracting the weight of lactose in the original lactose solution from the total weight. Additional lactose was then added to the lyophilised powder so that it contained equal weights of LG2055 and lactose. The LG2055 powder thus prepared contained $1.4 \times 10^{11}$ colony-forming units (cfu) of LG2055/g of powder, and was stored at $-30^{\circ} \mathrm{C}$. For the cell culture assay, washed LG2055 cells were lyophilised without lactose and stored at $-80^{\circ} \mathrm{C}$ until use.

\section{Mice and diets}

Male C57BL/6J mice, aged 7 weeks, purchased from Charles River Japan Inc., were housed individually in plastic cages in an air-conditioned specific pathogen-free room $\left(21-24^{\circ} \mathrm{C}\right.$, lights on $07.00-19.00$ hours) with free access to food and deionised water. The mice were acclimatised to a powder diet for 1 week, then divided into three groups (sixteen mice per group): those fed a normal-fat diet (NFD), a high-fat diet (HFD), or a high-fat diet containing LG2055 (HFD-LG).

Experimental diets were prepared according to the American Institute of Nutrition (AIN)-76 formula with some modifications ${ }^{(15,18)}$. Compositions of the experimental diets are shown in Table 1. These diets contained $20 \%$ skimmed milk, which is not used in the original AIN-76 formula. The protein and carbohydrate contents that were increased by the addition of skimmed milk were balanced by decreasing the amounts of casein and sucrose, respectively. The NFD contained $5 \%$ maize oil $(11.7 \%$ energy from fat $)$ as commonly described, and the HFD and HFD-LG contained $2 \%$ maize oil and $18 \%$ lard $39.2 \%$ energy from fat). The weight difference in the fat content among the diets was compensated for with sucrose. The HFD-LG contained $1 \%$ of the LG2055 powder to provide $1.4 \times 10^{9} \mathrm{cfu}$ of viable LG2055 cells with $0.5 \%$ lactose per $1 \mathrm{~g}$ of the diet. The NFD and HFD were supplemented with $0.5 \%$ lactose to equalise the lactose content among the diets. Mice were fed these diets and their body weight was monitored once a week. After 18 weeks of feeding, an intestinal permeability assay as described later was performed. At the end of the experimental period of 21 weeks, mice were fasted for $12 \mathrm{~h}$, anaesthetised with isoflurane, and exsanguinated via the portal vein. The serum prepared from the portal blood was stored at $-80^{\circ} \mathrm{C}$ until use. The liver and the mesenteric, perirenal/retroperitoneal and epididymal fat were removed from each mouse and weighed.

The protocol was submitted to, and approved by, the Animal Care and Use Committee of the Milk Science

Table 1. Diet composition

\begin{tabular}{|c|c|c|c|}
\hline \multirow[b]{2}{*}{ Ingredient } & \multicolumn{3}{|c|}{$(\%, w / w)$} \\
\hline & NFD* & HFD† & HFD-LG† \\
\hline Skimmed milk & $20 \cdot 0$ & $20 \cdot 0$ & $20 \cdot 0$ \\
\hline Casein & $12 \cdot 5$ & $12 \cdot 5$ & $12 \cdot 5$ \\
\hline DL-Methionine & 0.3 & 0.3 & 0.3 \\
\hline$\alpha$-Maize starch & $15 \cdot 0$ & $15 \cdot 0$ & $15 \cdot 0$ \\
\hline Sucrose & $37 \cdot 2$ & $22 \cdot 2$ & $21 \cdot 7$ \\
\hline Maize oil & $5 \cdot 0$ & $2 \cdot 0$ & $2 \cdot 0$ \\
\hline Lard & - & $18 \cdot 0$ & $18 \cdot 0$ \\
\hline Cellulose & $5 \cdot 0$ & $5 \cdot 0$ & $5 \cdot 0$ \\
\hline AIN-76 mineral mixture & 3.5 & 3.5 & 3.5 \\
\hline AIN-76 vitamin mixture§ & 1.0 & 1.0 & 1.0 \\
\hline Lactbacillus gasseri SBT2055 & - & - & 0.5 \\
\hline Lactose \| & 0.5 & 0.5 & 0.5 \\
\hline
\end{tabular}

NFD, normal-fat diet; HFD, high-fat diet; HFD-LG, high-fat diet containing LG2055; AIN, American Institute of Nutrition.

${ }^{*} 5 \%(\mathrm{w} / \mathrm{w})$ fat, $11.7 \%$ energy from fat.

$\dagger 20 \%(\mathrm{w} / \mathrm{w})$ fat, $39.2 \%$ energy from fat.

‡ HFD containing Lactobacillus gasseri SBT2055 $\left(1.4 \times 10^{9}\right.$ colony-forming units/g).

$\S$ AIN-76 vitamin mixture containing choline bitartrate.

II Lactose was used in the lyophilisation processes of LG2055 cells to reduce viability loss. 
Research Institute of Megmilk Snow Brand Co., Ltd, whose guidelines are based on those of the Science Council of Japan.

\section{Isolation of stromal-vascular fraction from epididymal fat}

Isolation of the stromal-vascular fraction (SVF) was performed as previously described ${ }^{(19)}$. Briefly, epididymal adipose tissues from mice were dissected and suspended in an ice-cold Roswell Park Memorial Institute (RPMI) medium (Gibco) supplemented with fetal bovine serum (FBS) and antibiotics, then transferred into PBS supplemented with $1 \mu \mathrm{g} / \mathrm{ml}$ heparin. Adipose tissues were then cut into fine pieces that were centrifuged. Floating pieces of adipose tissue were digested at $37^{\circ} \mathrm{C}$ for $20 \mathrm{~min}$ with $1 \mathrm{mg} / \mathrm{ml}$ collagenase (Sigma) and $10 \mu \mathrm{g} / \mathrm{ml}$ DNase I (Roche) in RPMI medium. The samples were passed through a $70 \mu \mathrm{m}$ mesh and centrifuged at $620 \mathrm{~g}$ for $3 \mathrm{~min}$. Pellets thus collected were re-suspended into an erythrocyte lysis buffer $\left(168 \mathrm{~mm}-\mathrm{NH}_{4} \mathrm{Cl}, 10 \mathrm{~mm}-\mathrm{KHCO}_{3}\right.$ and 0.08 mM-EDTA), incubated for $3 \mathrm{~min}$, and washed with an ice-cold flow cytometry buffer (FCM buffer; $1 \%$ FBS, $0.05 \%$ sodium azide in PBS) to obtain SVF.

\section{Flow cytometry analysis}

The SVF $\left(1 \times 10^{5-6}\right.$ cells per assay tube $)$ was washed with PBS and centrifuged at $620 \mathrm{~g}$ for $3 \mathrm{~min}$. Pellets were suspended with Zombie Aqua (BioLegend) live-dead discriminating dye, diluted with PBS, and incubated at room temperature for $15 \mathrm{~min}$ in the dark. Cells were washed with FCM buffer and fixed with $0.1 \%$ paraformaldehyde/PBS at room temperature for $20 \mathrm{~min}$ in the dark. Fixed cells were washed with FCM buffer, centrifuged and suspended in an anti-mouse CD16/32 (eBioscience)/FCM buffer, followed by incubation at $4^{\circ} \mathrm{C}$ for $10 \mathrm{~min}$ in the dark. Cells were washed with FCM buffer then stained with antibodies interacting with cell-surface antigens or matching control isotypes for $30 \mathrm{~min}$. The following antibodies were used: anti-mouse fluorescein isothiocyanate (FITC)-labelled CD11b (BD Bioscience), allophycocyaninlabelled CD11c (BD Bioscience), phycoerythrin-labelled CD206 (BD Bioscience), Brilliant Violet 421-labelled F4/80 (BioLegend), Pacific Blue-labelled CD4 (BD Bioscience) or FITC-labelled CD8a (eBioscience). The cells were rinsed and re-suspended in FCM buffer and analysed using a FACS Cant II flow cytometer (BD Bioscience). A cell population with CD11b-, F4/80- and CD11c-positive but CD206-negative was defined as a pro-inflammatory macrophage population (classically activated macrophages or M1 subtype), and that with CD11b-, F4/80- and CD206-positive but CD11c-negative was defined as an anti-inflammatory macrophage population (alternatively activated macrophages or M2 subtype). CD4positive but $\mathrm{CD} 8$-negative $\mathrm{T}$ cells were defined as $\mathrm{CD} 4$-positive $\mathrm{T}$ cell subpopulation and $\mathrm{CD} 8$-positive but $\mathrm{CD} 4$-negative $\mathrm{T}$ cells were defined as CD8-positive T cell subpopulation.

\section{In vivo intestinal permeability assay}

Intestinal permeability was measured as previously reported ${ }^{(20)}$ with some modifications. Mice fed experimental diets for 18 weeks were fasted for $12 \mathrm{~h}$ and orally given FITC-dextran with average molecular weight of 3000-5000 Da (FD-4) (Sigma) in saline $(500 \mathrm{mg} / \mathrm{kg}$ body weight, $125 \mathrm{mg} / \mathrm{ml}$ ). After 1 and $4 \mathrm{~h}, 100 \mu \mathrm{l}$ of blood were collected from the orbital sinus. Plasma was obtained by the addition of $10 \mu \mathrm{g} / \mathrm{ml}$ heparin and centrifugation at $1200 \mathrm{~g}$ for $30 \mathrm{~min}$ at $4^{\circ} \mathrm{C}$. The plasma was diluted 3-fold with PBS and analysed for FD-4 concentration with a fluorescence spectrophotometer (Varioskan Flash; Thermo Scientific) at an excitation wavelength of $485 \mathrm{~nm}$ and an emission wavelength of $535 \mathrm{~nm}$.

\section{Serum anti-lipopolysaccharide antibody assay}

Serum obtained from portal blood was analysed for anti-LPS IgG levels using a commercial ELISA kit from Chondrex.

\section{Cell culture}

Caco-2 cells were purchased from the American Type Culture Collection (ATCC). Dulbecco's modified Eagle's medium (DMEM) (Sigma) supplemented with 10\% FBS, 1\% nonessential amino acids and antibiotics was used as a growth medium. The cells were seeded into a twenty-four-well cell culture insert (BD Bioscience) $\left(5 \times 10^{4}\right.$ cells $\left./ \mathrm{cm}^{2}\right)$ that was pre-coated with collagen (Nitta Gelatin). After $21 \mathrm{~d}$, cells were differentiated into an epithelial cell-like phenotype and used for the following assay.

\section{Paracellular permeability assay using Caco-2 cell monolayers}

Paracellular permeability was determined by trans-epithelial electrical resistance (TEER) and the fluorescent dye flux across Caco- 2 cell monolayers. TEER was measured using Millicell ERS-2 (Millipore) and expressed in $\Omega \times \mathrm{cm}^{2}$. The fluorescent dye flux was measured using an impermeable fluorescent Lucifer yellow dye (LY; Invitrogen). LY (100 $\mu \mathrm{M})$ was added to the apical side of Caco-2 monolayers, and the concentration of LY infiltrated into the basal side of Caco-2 monolayers was measured after $3 \mathrm{~h}$ using a fluorescence spectrophotometer (Varioskan Flash; Thermo Scientific) at an excitation wavelength of $428 \mathrm{~nm}$ and an emission wavelength of $535 \mathrm{~nm}$. The LY flux was expressed in $\mathrm{pmol} / \mathrm{h} \times \mathrm{cm}^{2}$.

LG2055 cells $(1 \mathrm{mg} / \mathrm{ml})$ suspended in DMEM without FBS were added to the apical side of Caco-2 monolayers and interferon- $\gamma(\mathrm{IFN}-\boldsymbol{\gamma}, 50 \mathrm{ng} / \mathrm{ml}$; Pepro Tech) and TNF- $\alpha(10$ $\mathrm{ng} / \mathrm{ml}$, Pepro Tech) were simultaneously added to the basal side for $3 \mathrm{~d}$ to promote paracellular permeability. TEER was monitored daily and the LY flux was measured $72 \mathrm{~h}$ after treatment with LG2055 and the cytokines.

\section{Statistical analysis}

Data were expressed as mean values and standard deviations. Statistical analyses were performed by using PASW Statistics 17.0 (SPSS, Inc.) and StatView software package 5.0 (SAS Institute, Inc.). Data were analysed by one-way ANOVA, followed by Dunnett's test, which compared the NFD group or the HFD-LG group with the HFD group in in vivo experiments, and the control group or the IFN- $\gamma+$ TNF- $\alpha+$ LG2055 group 
with the IFN- $\gamma+$ TNF- $\alpha$ group in in vitro experiments. $P$ values $<0.05$ were considered statistically significant. Correlation analysis was assessed by the Pearson's test using StatView software.

\section{Results}

LG2055 prevents high-fat diet-induced weight gain of the body and adipose tissues

The quantity of the given diet consumed and the weights of the total body, adipose tissues and liver are shown in Table 2. Despite a smaller intake quantity in the HFD and HFD-LG groups than in the NFD group, energy intake was greater in the HFD and HFD-LG groups than in the NFD group, with no significant differences in either diet or energy intake between the HFD and HFD-LG groups. The HFD group exhibited significantly greater weights of the body, adipose tissues and liver than the NFD group. In a comparison between the HFD and HFD-LG groups, the HFD-LG group showed significantly lower weights of the body, mesenteric and perirenal/retroperitoneal adipose tissues than the HFD group. Thus, LG2055 prevented HFD-induced increases in the body and adipose tissue weights, despite the same energy intake by the HFD and HFD-LG groups.

\section{LG2055 prevents high-fat diet-augmented inflammatory status in epididymal adipose tissue}

The immune cell population in epididymal adipose tissue was analysed. There were no effects of LG2055 on the total number of stromal-vascular live cells (data not shown). The percentage of adipose tissue macrophages in stromal-vascular live cells was increased in the HFD group compared with the NFD group (Fig. 1(a)). The percentage of classically activated M1 macrophages, which have pro-inflammatory properties, was increased in the HFD group (Fig. 1(b)), while that of

Table 2. Diet intake, body weight, adipose tissue weight and liver weight $\dagger$ (Mean values and standard deviations; sixteen mice per group)

\begin{tabular}{|c|c|c|c|c|c|c|}
\hline \multirow[b]{3}{*}{ Parameters } & \multicolumn{6}{|c|}{ Experimental groups } \\
\hline & \multicolumn{2}{|c|}{ NFD } & \multicolumn{2}{|c|}{ HFD } & \multicolumn{2}{|c|}{ HFD-LG } \\
\hline & Mean & SD & Mean & SD & Mean & SD \\
\hline Average diet intake $(\mathrm{g} / \mathrm{d})$ & $3.06^{*}$ & 0.07 & $2 \cdot 86$ & 0.22 & $2 \cdot 87$ & 0.17 \\
\hline $\begin{array}{l}\text { Average energy intake } \\
\qquad(\mathrm{kJ} / \mathrm{d})\end{array}$ & $49 \cdot 17^{*}$ & $1 \cdot 12$ & 54.96 & $4 \cdot 17$ & $55 \cdot 11$ & 3.20 \\
\hline Initial body weight (g) & 23.91 & $1 \cdot 13$ & 23.92 & 1.62 & 23.93 & 1.30 \\
\hline Final body weight (g) & $32 \cdot 61^{*}$ & 1.94 & 42.04 & 4.67 & $39.08^{*}$ & $2 \cdot 11$ \\
\hline \multicolumn{7}{|c|}{ Adipose tissue weight (g) } \\
\hline Mesenteric & $0.51^{*}$ & $0 \cdot 10$ & 1.00 & 0.38 & $0.79^{*}$ & $0 \cdot 16$ \\
\hline Epididymal & $1.24^{*}$ & 0.40 & $2 \cdot 57$ & 0.47 & $2 \cdot 38$ & 0.51 \\
\hline $\begin{array}{l}\text { Perirenal/ } \\
\quad \text { retroperitoneal }\end{array}$ & $0.53^{\star}$ & 0.21 & 1.29 & 0.30 & $1.07^{*}$ & 0.24 \\
\hline Total & $2 \cdot 26^{\star}$ & 0.73 & 4.87 & 0.94 & 4.24 & 0.85 \\
\hline Liver weight (g) & $1.01^{*}$ & 0.06 & $1 \cdot 13$ & 0.23 & 1.03 & 0.12 \\
\hline
\end{tabular}

NFD, normal-fat diet; HFD, high-fat diet; HFD-LG, high-fat diet containing LG2055. ${ }^{*}$ Mean value was significantly different from that of the HFD group $(P<0.05)$.

†Data were analysed by one-way ANOVA, followed by Dunnett's multiplecomparison test. All parameters except 'Initial body weight' were determined 21 weeks after the start of feeding the experimental diets. alternatively activated M2 macrophages, which have antiinflammatory properties, was not affected by HFD (Fig. 1 (c)). The M1:M2 ratio, which represents the degree of inflammatory status in adipose tissue, was increased by the HFD (Fig. 1(d)). Compared with the HFD group, the HFD-LG group showed a significantly larger proportion of antiinflammatory M2 macrophages (Fig. 1(c)) and a significantly lower M1:M2 ratio (Fig. 1(d)), suggesting that the intake of the HFD-LG ameliorates an HFD-induced disposition in macrophage composition to a pro-inflammatory status.

We also analysed the $\mathrm{T}$ cell subpopulation, which is involved in inflammation in adipose tissue. The HFD reduced the CD4-positive $\mathrm{T}$ cell subpopulation in adipose tissue compared with the NFD (Fig. 1(e)), while the CD8-positive $\mathrm{T}$ cells (Fig. 1(f)) did not significantly differ between the HFD and NFD groups. Compared with the HFD group, the HFD-LG group had a decreased proportion of CD8-positive cells (Fig. 1(f)), suggesting that LG2055 modulated part of the $\mathrm{T}$ cell subpopulation in the adipose tissue.

Taken together, these results suggest that LG2055 could prevent the inflammation in the adipose tissue caused by HFD.

\section{LG2055 prevents high-fat diet-induced intestinal permeability}

We analysed the intestinal permeability to FD-4, a fluoresceinlabelled dextran, in mice fed the experimental diets for 18 weeks. The HFD group showed a higher plasma concentration of FD-4 at $1 \mathrm{~h}$ after oral administration of FD-4 than the NFD group, indicating disruption of the intestinal barrier by the HFD (Fig. 2(a)). In contrast, at the same time point, the plasma FD-4 concentration in the HFD-LG group was lower than that in the HFD group, and the concentration was approximately equal to that in the NFD group (Fig. 2 (a)). The FD-4 area under the concentration-time curve (AUC $0-4 \mathrm{~h}$ ) also showed a significantly lower value in the HFD-LG group compared with the HFD group (Fig. 2(b)). Thus, LG2055 was shown to prevent the increase in the intestinal permeability caused by the HFD.

To examine whether the protective effect of LG2055 on the intestinal barrier affects the entry of bacterial LPS into the portal vein, we analysed anti-LPS IgG levels in the blood, which reflect the host immune response to bacterial LPS. The levels were increased by the HFD compared with the NFD, and this increase was suppressed by LG2055 supplementation to the diet (Fig. 2(c)), implying the protective effect of LG2055 against the entry of bacterial LPS.

To identify whether the changes in gut permeability and anti-LPS IgG levels in the blood are associated with the adipose tissue inflammation, multiple correlation analysis between these parameters was performed. The M1:M2 ratio in adipose tissue was positively correlated with plasma FD-4 and anti-LPS IgG levels (Fig. 2(d) and (e)). In addition, there was a positive correlation between plasma FD-4 and anti-LPS IgG levels (Fig. 2(f)). These results suggest that the changes in gut permeability relate to adipose tissue inflammation. 
(a)

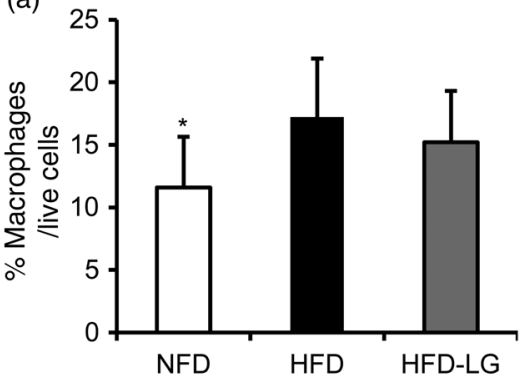

(c)

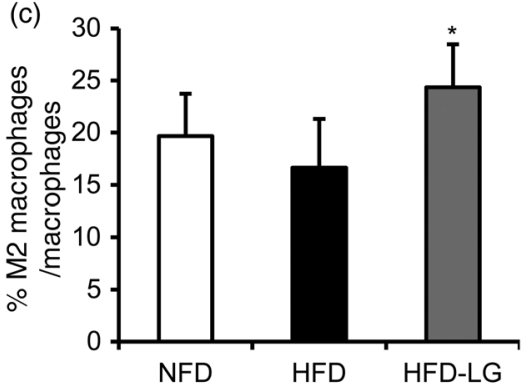

(e)

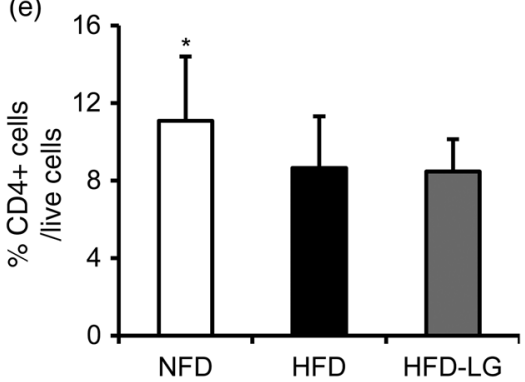

(b)

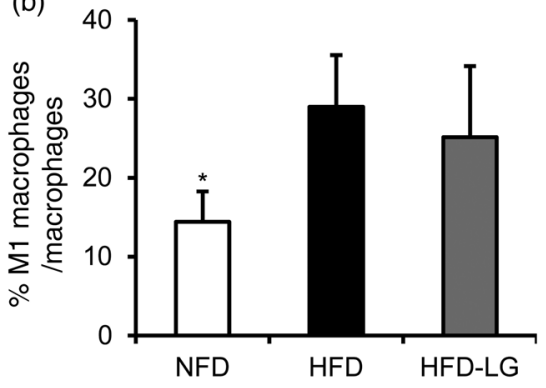

(d)

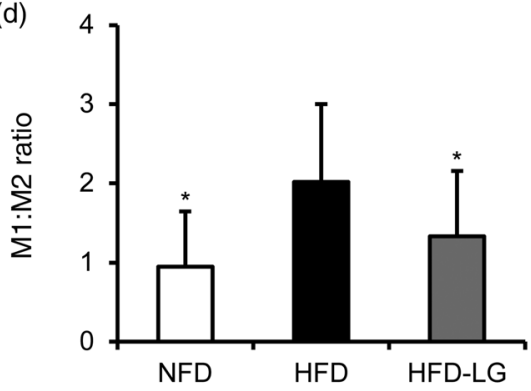

(f)

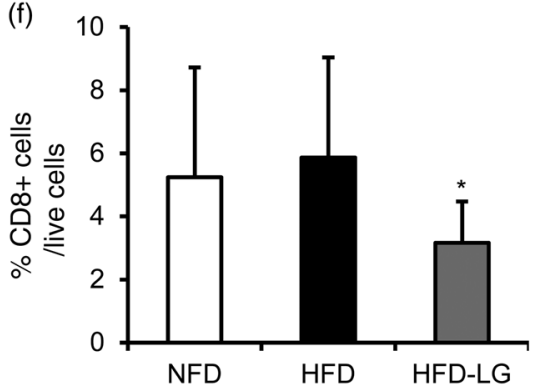

Fig. 1. Effect of Lactobacillus gasseri SBT2055 (LG2055) on immune cell populations in epididymal adipose tissue. The stromal-vascular fraction (SVF) was prepared from epididymal fat tissue of mice fed a normal-fat diet (NFD), high-fat diet (HFD) or the HFD containing LG2055 (HFD-LG) for 21 weeks, and cells in the SVF were analysed by flow cytometry. (a) Percentage of F4/80- and CD11b-positive cells defined as macrophages in live cells of the SVF. Percentages of CD11c-positive and CD206-negative macrophages defined as classically activated (M1) macrophages (b), and CD11c-negative and CD206-positive macrophages defined as alternatively activated (M2) macrophages (c) in total macrophages. (d) Ratio of M1 macrophages to M2 macrophages. (e) Percentage of CD4-positive cells in live cells of the SVF. (f) Percentage of CD8-positive cells in live cells of the SVF. Values are means, with standard deviations represented by vertical bars ( $n$ 15-16 in each group). Data were analysed by one-way ANOVA, followed by Dunnett's multiple-comparison test. * Mean value was significantly different from that of the HFD group $(P<0.05)$.

\section{LG2055 prevents paracellular permeability increased by pro-inflammatory cytokines in Caco-2 cell monolayers}

As described above, LG2055 consumption prevented the increase in the intestinal permeability caused by the HFD in mice. We further examined the preventive effect of LG2055 on the barrier function using Caco-2 cell monolayers. Differentiated Caco-2 cells grown on a permeable support were basally treated with IFN- $\gamma$ and TNF- $\alpha$ to induce impairment of the intestinal barrier for $72 \mathrm{~h}$. We monitored TEER values every $24 \mathrm{~h}$ and paracellular permeability to LY across the Caco- 2 monolayers after $72 \mathrm{~h}$. When Caco- 2 cell monolayers were treated with these cytokines, the TEER value was significantly reduced at $72 \mathrm{~h}$ (Fig. 3(a)) and the permeability to LY was significantly increased (Fig. 3(b)) compared with control without cytokines. The reduction in TEER and increase in LY permeability were both significantly ameliorated by the apical treatment with LG2055.
These results suggest that LG2055 can directly affect epithelial cells to improve their barrier function.

\section{Discussion}

A previous study showed that mice fed a HFD containing LG2055 cells had lower body and abdominal adipose tissue weights, and that consumption of LG2055 cells prevented the elevation of mRNA expression of pro-inflammatory markers Ccl2, Ccr2, and Tnf in adipose tissue ${ }^{(15)}$. Adipose tissue inflammation is initiated by the infiltration of macrophages and $T$ cells into adipose tissue. On the other hand, recent studies suggested the disruption of intestinal barrier as a cause of inflammation in obesity ${ }^{(4,21)}$. These observations prompted us to investigate the effect of LG2055 on the immune cell population in adipose tissue and the barrier function of the intestine. Our present study demonstrated that mice fed a HFD 


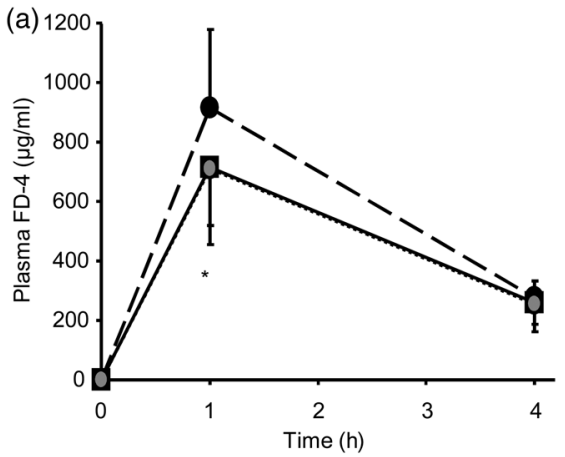

(b)
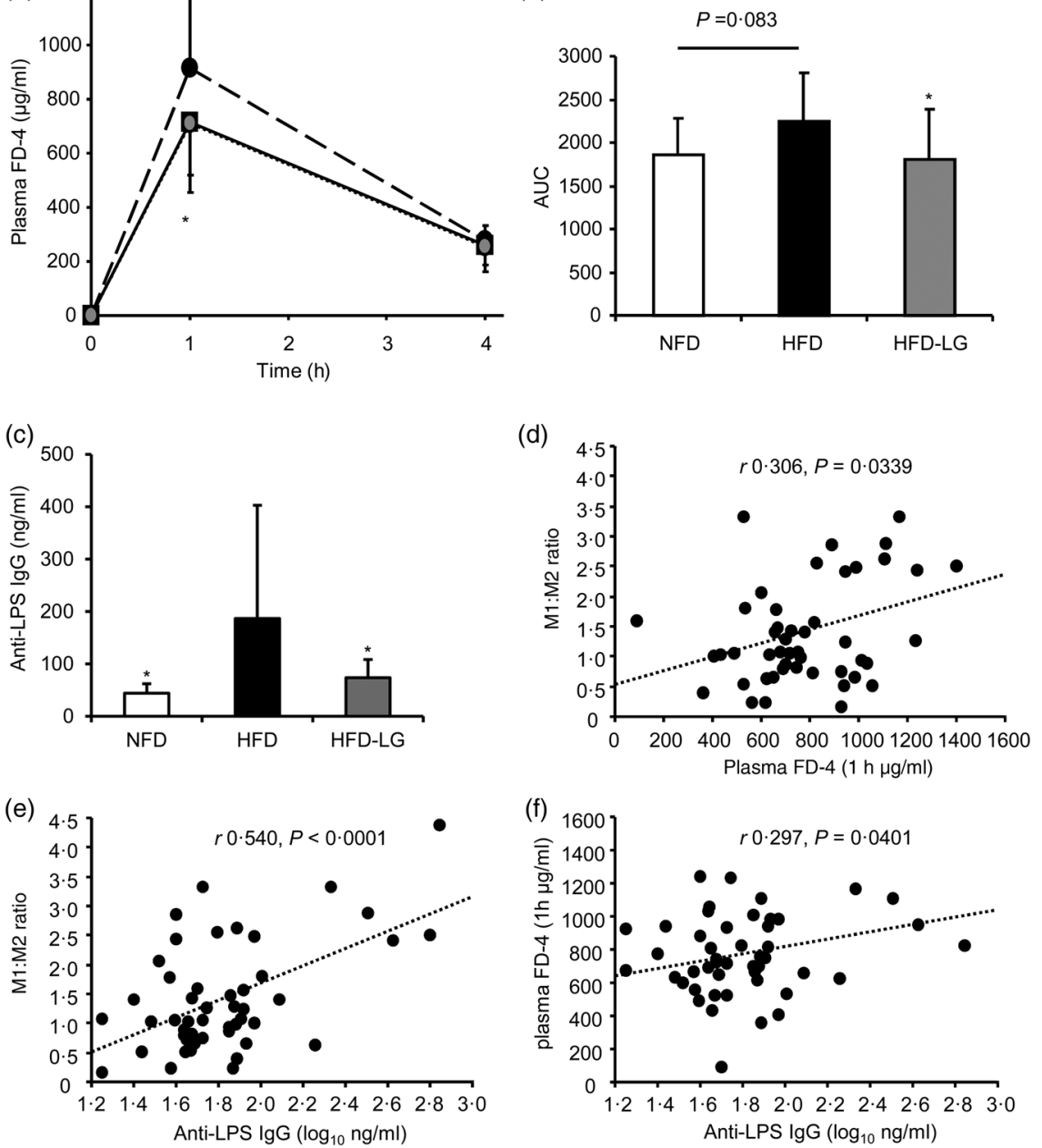

Fig. 2. Effect of Lactobacillus gasseri SBT2055 (LG2055) on intestinal permeability and anti-lipopolysaccharide (LPS) antibody response. (a) Plasma fluorescein isothiocyanate-dextran (FD-4) levels were measured 1 and $4 \mathrm{~h}$ after oral administration of FD-4 in mice fed a normal-fat diet (NFD; - $\square-$ ), a high-fat diet (HFD; --) or the HFD containing LG2055 (HFD-LG; -----) for 18 weeks. (b) AUC of plasma FD-4 levels in each group. (c) Anti-LPS IgG levels in the serum obtained from the portal vein. Values are means, with standard deviations represented by vertical bars ( $n 16$ in each group). Data were analysed by one-way ANOVA, followed by Dunnett's multiple-comparison test. * Mean value was significantly different from that of the HFD group $(P<0.05)$. Correlations between the plasma FD-4, anti-LPS antibody and adipose M1:M2 ratio parameters were plotted $(\mathrm{d}-\mathrm{f})$ with Pearson's $r$ correlation and the corresponding $P$ value.

$(20 \%(\mathrm{w} / \mathrm{w}))$ had a remarkable gain in body weight and visceral fat mass compared with those fed a normal-fat $(5 \%$ (w/w)) diet (NFD), whereas a HFD containing LG2055 (HFD-LG) significantly suppressed the gain in body weight and mesenteric and perirenal/retroperitoneal adipose tissue weights. Thus, we initially confirmed that the present study successfully reached a state of diet-induced obesity and LG2055 improved the status, giving the rationale for the succeeding evaluation about whether LG2055 influences inflammatory status in adipose tissue and the intestinal barrier function.

The abundance of activated macrophages is a typical status of inflammatory adipose tissue and activated macrophages are classified into two groups: classically activated macrophages (M1) that produce pro-inflammatory cytokines such as TNF- $\alpha$ and IL- 6 , and alternatively activated macrophages (M2) that express anti-inflammatory markers such as arginase-1 and IL-10. While M2 macrophages are dominant in lean adipose tissue, the percentage of M1 macrophages increases as obesity progresses ${ }^{(22)}$. Thus, the balance between pro-inflammatory M1 and anti-inflammatory M2 macrophages plays an important role in the development of adipose tissue inflammation. The removal of CD11c-positive cells that include M1 macrophages remedies adipose tissue inflammation as well as insulin resistance in an obese mouse model $^{(23)}$. Furthermore, macrophage-specific disruption of the PPAR- $\gamma$ gene, which is required for the activation of M2 macrophages, aggravates adipose tissue inflammation and insulin resistance in diet-induced obese mice ${ }^{(24)}$, suggesting that M2 macrophages have protective effects against inflammation. In addition to macrophages, effector $\mathrm{T}$ cells are also involved in the progress of adipose tissue inflammation in obesity. A smaller number of CD4-positive T cells and a larger number of $\mathrm{CD} 8$-positive $\mathrm{T}$ cells were observed in the adipose tissue of obese mice compared with that of lean mice ${ }^{(25,26)}$. Nishimura et al. reported that immunological and genetic depletion of CD8-positive cells prevented adipose tissue inflammation and the development of systemic insulin 

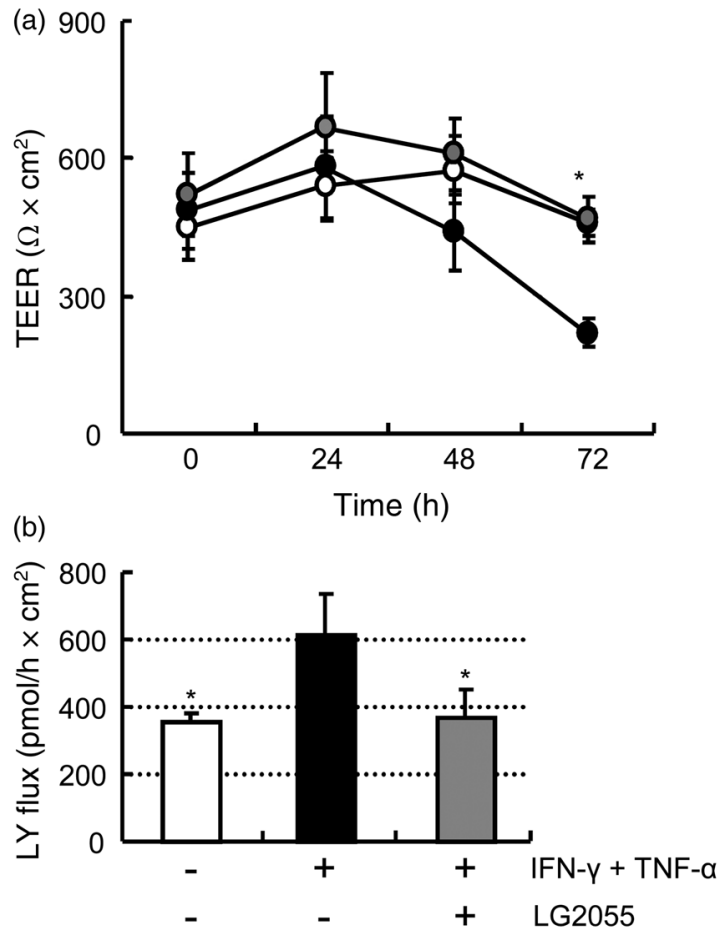

Fig. 3. Effect of Lactobacillus gasseri SBT2055 (LG2055) on paracellular permeability of Caco-2 cell monolayers. Caco-2 cell monolayers were treated with $1 \mathrm{mg} / \mathrm{ml}$ LG2055 (apically), and $50 \mathrm{ng} / \mathrm{ml}$ interferon- $\gamma$ (IFN- $\gamma$ ) and $10 \mathrm{ng} / \mathrm{ml}$ TNF- $\alpha$ (basally) for $72 \mathrm{~h}$. (a) Trans-epithelial electric resistance (TEER) was measured $0,24,48$ and $72 \mathrm{~h}$ after the treatment. $-\mathrm{O}-$, Control; -- -, IFN- $\gamma+$ TNF- $\alpha$; - - , IFN- $\gamma+$ TNF- $\alpha+$ LG2055. (b) Rate of the Lucifer yellow (LY) flux across Caco- 2 monolayers was measured $72 \mathrm{~h}$ after the treatment. Values are means, with standard deviations represented by vertical bars ( $n 3$ in each group). Data were analysed by one-way ANOVA, followed by Dunnett's multiple-comparison test. * Mean value was significantly different from that of the IFN- $\gamma+$ TNF- $\alpha$ group $(P<0.05)$.

resistance $^{(25)}$. In the present study, HFD intake induced significant increases in M1 macrophages and the M1:M2 ratio, but a significant reduction in CD4-positive cells in adipose tissue. These results are consistent with observations in other studies on HFD-induced obese mice that showed an increase in M1 macrophages and a decrease in CD4-positive T cells in adipose tissue ${ }^{(25,27,28)}$. Such alterations generated by the HFD were modified by the consumption of LG2055 (HFD-LG), resulting in a significant increase in M2 macrophages and a significant decrease in the M1:M2 ratio in adipose tissue. Furthermore, the HFD-LG induced a significant reduction in CD8-positive cells in the tissue. Thus, LG2055 prevented the immune cell population in adipose tissue from being disposed towards inflammatory characteristics.

M2 macrophages are known to be induced by IL-4 and IL-13 ${ }^{(29)}$ as well as by IL-10 ${ }^{(30)}$. As LG2055 has been found to stimulate the IL-10 production of dendritic cells ${ }^{(31)}$, this might be partly responsible for the augmentation of M2-type macrophages by LG2055. It has also been reported that $L$. paracasei and L. rhamnosus prevented an increase in M1 macrophage markers (CD11c, MMP12) in adipose tissue during obesity $^{(32)}$, and some lactobacilli ameliorated intestinal inflammation by polarising the macrophage population into M2 macrophages in the colon ${ }^{(33,34)}$. The present study also reports that L. gasseri is the first of these species shown to increase M2 macrophages in adipose tissue.

Disturbance of the intestinal barrier function is involved in adipose tissue inflammation in obesity ${ }^{(4,35)}$. An important function of the intestinal barrier is to prevent pro-inflammatory molecules, such as LPS of intestinal bacteria origin, from entering the systemic circulation. When the intestinal barrier is disrupted by HFD intake or genetic obesity, plasma LPS levels are elevated and play a critical role in developing systemic low-grade inflammation in obesity ${ }^{(4,36,37)}$. In our study, mice fed the HFD showed significantly higher levels of FITC-dextran (FD-4), which is poorly absorbed from the intestine, in plasma compared with mice fed NFD. The plasma FD-4 levels peaked at $1 \mathrm{~h}$ and then declined by $4 \mathrm{~h}$, which is similar to other studies: for example, Cani et al. reported that FD-4-administered $\mathrm{Ob} / \mathrm{Ob}$ mice showed that plasma FD-4 peaked at $1 \mathrm{~h}$ and then decreased ${ }^{(20)}$. Furthermore, an elevation in LPS-specific antibody levels was observed in the serum of mice fed the HFD, implying an increased entry of LPS across the gut barrier. By contrast, mice fed the HFD containing LG2055 (HFD-LG) showed significantly lower levels of FD-4 and anti-LPS antibodies in the blood compared with mice fed the HFD. These results imply that LG2055 prevented the intestinal barrier disruption and the entry of LPS across the gut epithelial barrier caused by HFD intake.

Intestinal barrier function is often evaluated using Caco-2 cell monolayers. Bifidobacterium animalis ssp. lactis 420, for instance, is able to enhance the tight junction barrier of Caco-2 monolayers ${ }^{(38)}$, which may be a mechanism for the prevention of the entry of LPS into the blood ${ }^{(39)}$. We evaluated whether LG2055 enhances the tight junction barrier using Caco-2 monolayers. The monolayers, basally treated with pro-inflammatory cytokines IFN- $\gamma$ and TNF- $\alpha$ that are reported to be increased in plasma and intestine under obese or HFD-fed conditions ${ }^{(35,40)}$, increased their paracellular permeability, which was prevented by LG2055 cells apically added to the monolayers, suggesting that LG2055 cells enhanced the tight junction barrier. To further examine the improved tight junction barrier, we measured the expression levels of typical tight junction proteins (ZO-1, occludin and claudin-4) in Caco-2 cells as well as in the intestinal epithelial cells prepared from mice fed LG2055 by Western blotting. However, no effects of LG2055 on the tight junction proteins were observed in either sample (data not shown). The integrity of the tight junction is regulated by not only the expression levels but also the distribution of tight junction proteins (localisations of tight junction proteins to the plasma membranes or to the cytosol) ${ }^{(41)}$. For example, L. plantarum, which belongs to the same genus as LG2055, attenuated the dislocation of ZO-1 and occludin and prevented an increase in the paracellular permeability of Caco- 2 monolayers treated with phorbol ester $^{(42)}$. We also consider it important to further examine whether LG2055 enhances the barrier function through improving the distribution of the tight junction proteins.

In conclusion, LG2055 prevented body-weight gain, visceral fat accumulation and polarisation of macrophages into an inflammatory subtype in adipose tissue in mice fed a HFD. 
Consumption of LG2055 also prevented high-fat-induced increases in intestinal permeability and anti-LPS antibody levels in the blood, implying an inhibited entry of LPS across the gut epithelial barrier. Addition of LG2055 cells to Caco-2 cell monolayers inhibited paracellular permeability elevated by inflammatory cytokines. Thus, the enhancement of intestinal barrier function may reduce the entry of inflammatory substances such as LPS from the intestine into the circulation, contributing to the inhibition of adipose tissue inflammation and obesity.

\section{Acknowledgements}

The authors are grateful to Ken Ukibe, Maya Yamashita, Kurumi Takagi, Tomohiro Hosoya and Satoshi Higurashi (Megmilk Snow Brand Co. Ltd) for their skilful collaboration and useful suggestions. The authors also appreciate the work of Emiko Yamaguchi (YBS Co. Ltd) in preparing the experimental diets and technical assistance, and Masaki Kumai, Shigeki Kanoh and Mie Kasuga (YBS Co. Ltd) for animal care.

The present study was funded by Megmilk Snow Brand Co., Ltd. The funder provided support in the form of salaries for all authors. All authors are employed by Megmilk Snow Brand Co., Ltd. The content of this paper was neither influenced nor constrained by the fact.

All authors designed the study; M. K., M. M. and A. O. carried out experimental work; M. K. analysed data and wrote the manuscript; F. S. supervised the study and Y. K. reviewed the manuscript. All authors read and approved the final manuscript.

\section{References}

1. Gregor MF \& Hotamisligil GS (2011) Inflammatory mechanisms in obesity. Annu Rev Immunol 29, 415-445.

2. Johnson AMF \& Olefsky JM (2013) The origins and drivers of insulin resistance. Cell 152, 673-684.

3. Cani PD, Amar J, Iglesias MA, et al. (2007) Metabolic endotoxemia initiates obesity and insulin resistance. Diabetes 56, 1761-1772.

4. Cani PD, Bibiloni R, Knauf C, et al. (2008) Changes in gut microbiota control metabolic endotoxemia-induced inflammation in high-fat diet-induced obesity and diabetes in mice. Diabetes 57, 1470-1481.

5. Nishida J, Ekataksin W, McDonnell D, et al. (1994) Ethanol exacerbates hepatic microvascular dysfunction, endotoxemia, and lethality in septic mice. Shock 1, 413-418.

6. Gäbele E, Dostert K, Hofmann C, et al. (2011) DSS induced colitis increases portal LPS levels and enhances hepatic inflammation and fibrogenesis in experimental NASH. I Hepatol 55, 1391-1399.

7. Mazzon E \& Cuzzocrea S (2008) Role of TNF- $\alpha$ in ileum tight junction alteration in mouse model of restraint stress. Am J Physiol Gastrointest Liver Physiol 294, G1268-G1280.

8. Moreira APB, Alves RDM, Teixeira TFS, et al. (2015) Higher plasma lipopolysaccharide concentrations are associated with less favorable phenotype in overweight/obese men. Eur J Nutr 54, 1363-1370.

9. Trøseid M, Nestvold TK, Rudi K, et al. (2013) Plasma lipopolysaccharide is closely associated with glycemic control and abdominal obesity: evidence from bariatric surgery. Diabetes Care 36, 36273632.

10. Fujiwara S, Seto Y, Kimura A, et al. (2001) Establishment of orallyadministered Lactobacillus gasseri SBT2055SR in the gastrointestinal tract of humans and its influence on intestinal microflora and metabolism. I Appl Microbiol 90, 343-352.

11. Takahashi H, Fujita T, Suzuki Y, et al. (2006) Monitoring and survival of Lactobacillus gasseri SBT2055 in the human intestinal tract. Microbiol Immunol 50, 867-870.

12. Usman, Hosono A (1999) Bile tolerance, taurocholate deconjugation, and binding of cholesterol by Lactobacillus gasseri strains. J Dairy Sci 82, 243-248.

13. Kadooka Y, Sato M, Imaizumi K, et al. (2010) Regulation of abdominal adiposity by probiotics (Lactobacillus gasseri SBT2055) in adults with obese tendencies in a randomized controlled trial. Eur J Clin Nutr 64, 636-643.

14. Kadooka Y, Sato M, Ogawa A, et al. (2013) Effect of Lactobacillus gasseri SBT2055 in fermented milk on abdominal adiposity in adults in a randomised controlled trial. Br J Nutr 110, 1696-1703.

15. Miyoshi M, Ogawa A, Higurashi S, et al. (2014) Anti-obesity effect of Lactobacillus gasseri SBT2055 accompanied by inhibition of pro-inflammatory gene expression in the visceral adipose tissue in diet-induced obese mice. Eur J Nutr 53, 599-606.

16. Sato M, Uzu K, Yoshida T, et al. (2008) Effects of milk fermented by Lactobacillus gasseri SBT2055 on adipocyte size in rats. Br J Nutr 99, 1013-1017.

17. Kadooka Y, Ogawa A, Ikuyama K, et al. (2011) The probiotic Lactobacillus gasseri SBT2055 inhibits enlargement of visceral adipocytes and upregulation of serum soluble adhesion molecule (sICAM-1) in rats. Int Dairy J 21, 623-627.

18. American Institute of Nutrition (1977) Report of the American Institute of Nutrition ad hoc Committee on Standards for Nutritional Studies. J Nutr 107, 1340-1348.

19. Ukibe K, Miyoshi M \& Kadooka Y (2015) Administration of Lactobacillus gasseri SBT2055 suppresses macrophage infiltration into adipose tissue in diet-induced obese mice. Br J Nutr 114, $1180-1187$.

20. Cani PD, Possemiers S, Van de Wiele T, et al. (2009) Changes in gut microbiota control inflammation in obese mice through a mechanism involving GLP-2-driven improvement of gut permeability. Gut 58, 1091-1103.

21. Suzuki T \& Hara H (2010) Dietary fat and bile juice, but not obesity, are responsible for the increase in small intestinal permeability induced through the suppression of tight junction protein expression in LETO and OLETF rats. Nutr Metab (Lond) 7, 19.

22. Lumeng CN, Bodzin JL \& Saltiel AR (2007) Obesity induces a phenotypic switch in adipose tissue macrophage polarization. J Clin Invest 117, 175-184.

23. Patsouris D, Li PP, Thapar D, et al. (2008) Ablation of CD11c-positive cells normalizes insulin sensitivity in obese insulin resistant animals. Cell Metab 8, 301-309.

24. Odegaard JI, Ricardo-Gonzalez RR, Goforth MH, et al. (2007) Macrophage-specific PPAR $\gamma$ controls alternative activation and improves insulin resistance. Nature 447, 1116-1120.

25. Nishimura S, Manabe I, Nagasaki M, et al. (2009) CD8+ effector T cells contribute to macrophage recruitment and adipose tissue inflammation in obesity. Nat Med 15, 914-920.

26. Rausch ME, Weisberg S, Vardhana P, et al. (2008) Obesity in $\mathrm{C} 57 \mathrm{BL} / 6 \mathrm{~J}$ mice is characterized by adipose tissue hypoxia and cytotoxic T-cell infiltration. Int J Obes (Lond) 32, 451-463.

27. Fujisaka S, Usui I, Bukhari A, et al. (2009) Regulatory mechanisms for adipose tissue M1 and M2 macrophages in diet-induced obese mice. Diabetes 58, 2574-2582.

28. Shaul ME, Bennett G, Strissel KJ, et al. (2010) Dynamic, M2-like remodeling phenotypes of $\mathrm{CD} 11 \mathrm{c}+$ adipose tissue macrophages during high-fat diet-induced obesity in mice. Diabetes 59, 11711181.

29. Odegaard JI \& Chawla A (2011) Alternative macrophage activation and metabolism. Annu Rev Pathol Mecb Dis 6, 275-297.

30. Sica A \& Mantovani A (2012) Macrophage plasticity and polarization: in vivo veritas. J Clin Invest 122, 787-795.

31. Sakai F, Hosoya T, Ono-Ohmachi A, et al. (2014) Lactobacillus gasseri SBT2055 induces TGF- $\beta$ expression in dendritic cells and activates 
TLR2 signal to produce IgA in the small intestine. PLOS ONE 9, e105370.

32. Wang J, Tang H, Zhang C, et al. (2015) Modulation of gut microbiota during probiotic-mediated attenuation of metabolic syndrome in high fat diet-fed mice. ISME J 9, 1-15.

33. Jang SE, Han MJ, Kim SY, et al. (2014) Lactobacillus plantarum CLP-0611 ameliorates colitis in mice by polarizing M1 to M2-like macrophages. Int Immunopharmacol 21, 186-192.

34. Jang SE, Hyam SR, Han MJ, et al. (2013) Lactobacillus brevis G-101 ameliorates colitis in mice by inhibiting NF- $\mathrm{B}$, MAPK and AKT pathways and by polarizing M1 macrophages to M2-like macrophages. J Appl Microbiol 115, 888-896.

35. Lam YY, Ha CWY, Campbell CR, et al. (2012) Increased gut permeability and microbiota change associate with mesenteric fat inflammation and metabolic dysfunction in diet-induced obese mice. PLOS ONE 7, e34233.

36. Amar J, Chabo C, Waget A, et al. (2011) Intestinal mucosal adherence and translocation of commensal bacteria at the early onset of type 2 diabetes: molecular mechanisms and probiotic treatment. EMBO Mol Med 3, 559-572.
37. Schertzer JD, Tamrakar AK, Magalhães JG, et al. (2011) NOD1 activators link innate immunity to insulin resistance. Diabetes $\mathbf{6 0}$, 2206-2215.

38. Putaala H, Salusjärvi T, Nordström M, et al. (2008) Effect of four probiotic strains and Escherichia coli $\mathrm{O} 157: \mathrm{H} 7$ on tight junction integrity and cyclo-oxygenase expression. Res Microbiol 159, 692-698.

39. Stenman LK, Waget A, Garret C, et al. (2014) Potential probiotic Bifidobacterium animalis ssp. lactis 420 prevents weight gain and glucose intolerance in diet-induced obese mice. Benef Microbes 5, 437-445.

40. Li Z, Soloski MJ, Diehl AM, et al. (2005) Dietary factors alter hepatic innate immune system in mice with nonalcoholic fatty liver disease. Hepatology 42, 880-885.

41. Ulluwishewa D \& Anderson R (2011) Regulation of tight junction permeability by intestinal bacteria and dietary components. J Nutr 141, 769-776

42. Karczewski J, Troost FJ, Konings I, et al. (2010) Regulation of human epithelial tight junction proteins by Lactobacillus plantarum in vivo and protective effects on the epithelial barrier. Am J Physiol Gastrointest Liver Physiol 298, G851-G859. 